\title{
Current reversals in a rocking ratchet: Dynamical versus symmetry-breaking mechanisms
}

\author{
D. Cubero, ${ }^{1}$ V. Lebedev, ${ }^{2, *}$ and F. Renzoni ${ }^{2}$ \\ ${ }^{1}$ Departamento de Física Aplicada I, EUP, Universidad de Sevilla, Calle Virgen de África 7, 41011 Sevilla, Spain \\ ${ }^{2}$ Department of Physics and Astronomy, University College London, Gower Street, London WC1E 6BT, United Kingdom
}

(Received 17 May 2010; published 19 October 2010)

\begin{abstract}
Directed transport in ratchets is determined by symmetry breaking in a system out of equilibrium. A hallmark of rocking ratchets is current reversals: an increase in the rocking force changes the direction of the current. In this work for a biharmonically driven spatially symmetric rocking ratchet we show that a class of current reversal is precisely determined by symmetry breaking, thus creating a link between dynamical and symmetry-breaking mechanisms.
\end{abstract}

DOI: 10.1103/PhysRevE.82.041116

PACS number(s): 05.60.-k, 05.40.-a, 05.45.-a

Many processes in physics, chemistry, and biology involve directed transport through periodic structures. For the equilibrium situation of Brownian motion, diffusion can be turned into directed diffusion by the application of a dc bias. In out-of-equilibrium systems, new mechanisms for directed transport may arise. Counterintuitively, far from equilibrium it is possible to obtain directed transport through a macroscopically flat potential in the absence of an applied dc bias. This is the so-called ratchet effect [1-7].

The archetypal of a ratchet device is the rocking ratchet. In this setup, Brownian particles experience an asymmetric sawtooth potential and a sinusoidal rocking force. The rocking force drives the system out of equilibrium, and directed transport is generated following the breaking of the symmetries of the system. An analogous effect can also be produced in a spatially symmetric potential and a biharmonic force, with the latter playing the double role of driving the system out of equilibrium and breaking the relevant time symmetries [8-16].

A hallmark of rocking ratchets is current reversals. By progressively increasing the rocking force from zero, the generation of a current is observed, whose magnitude is first an increasing function of the strength of the driving. However, at larger values of the rocking force the current reaches a maximum, then decreases to zero, and changes sign. This feature can appear several times in a given system for different values of the force, thus producing multiple current reversals. Single and multiple current reversals have been observed in a variety of systems, both for an asymmetric potential and a symmetric drive and for a symmetric potential and a time-asymmetric drive [17-19].

Current reversals are usually considered a dynamical effect, not related to the symmetry breaking required to allow directed motion. In this work, for the specific system with a spatially symmetric potential and a time-asymmetric drive, we show that a class of current reversal is actually determined by dissipation-induced symmetry breaking. As a consequence, these reversals are not present in the Hamiltonian limit or in the overdamped limit.

Our work consists of a theoretical analysis of the relation-

\footnotetext{
*Present address: Institut für Quantenoptik, Leibnitz Universität Hannover, Welfengarten 1, D-30167 Hannover, Germany.
}

ship between current reversals and dissipation-induced symmetry breaking. This is carried out comparing differing regimes: weakly damped, Hamiltonian, and overdamped. In the case of weak damping, where current reversals associated with dissipative effects are present, the theoretical analysis is also supported by experimental results obtained with cold atom ratchets. In our theoretical analysis, the dynamics of particles in the considered spatially symmetric rocking ratchet is described by the Langevin equation

$$
m \ddot{x}=-\alpha \dot{x}-U^{\prime}(x)+F(t)+\xi(t),
$$

where $U(x)=U_{0} \cos (2 k x) / 2$ is a periodic potential, $\alpha$ is the friction coefficient, $\xi(r)$ is a Gaussian white noise: $\langle\xi(t)\rangle=0$, $\left\langle\xi(t) \xi\left(t^{\prime}\right)\right\rangle=2 D \delta\left(t-t^{\prime}\right)$, and $F(t)$ is an applied biharmonic drive of the form [20]

$$
F(t)=F_{0}[A \cos (\omega t)+B \cos (2 \omega t+\phi)] .
$$

The generation of a current in such out-of-equilibrium setup can be understood within the framework of the symmetry analysis [13-15]. For the considered spatially symmetric potential there are two time symmetries which need to be broken to allow for the generation of a current: the shift symmetry, which corresponds to invariance under the transformation $(x, p, t) \rightarrow(-x,-p, t+T / 2)$, with $T$ as the period of the drive, and the time-reversal symmetry, which requires invariance under the transformation $(x, p, t) \rightarrow(x,-p,-t)$. For a biharmonic drive of the form of Eq. (2), the shift symmetry is broken independently of the value of $\phi$ (for $A \neq 0$, $B \neq 0$ ). The breaking of the time-reversal symmetry depends on both the value of the phase $\phi$ and the dissipation level. In the Hamiltonian (dissipationless) case, the system is invariant under time reversal for $\phi=n \pi$, with $n$ as integer. Thus, for these values of the relative phase, no current can be generated. However, for nonzero weak dissipation the timereversal symmetry is broken by dissipation, and directed motion can be produced also for $\phi=n \pi$. In the regime of weak dissipation, the dependence of the particles' velocity $v$ on the phase $\phi$ is well described, in leading order, by $v$ $=v_{\max } \sin \left(\phi-\phi_{0}\right)$, where $\phi_{0}$ is determined by dissipation, and vanishes in the Hamiltonian limit. It has been shown recently that this sinelike functional form is a consequence solely of the system symmetries, being independent of the interaction details [21]. Of importance for the present study 


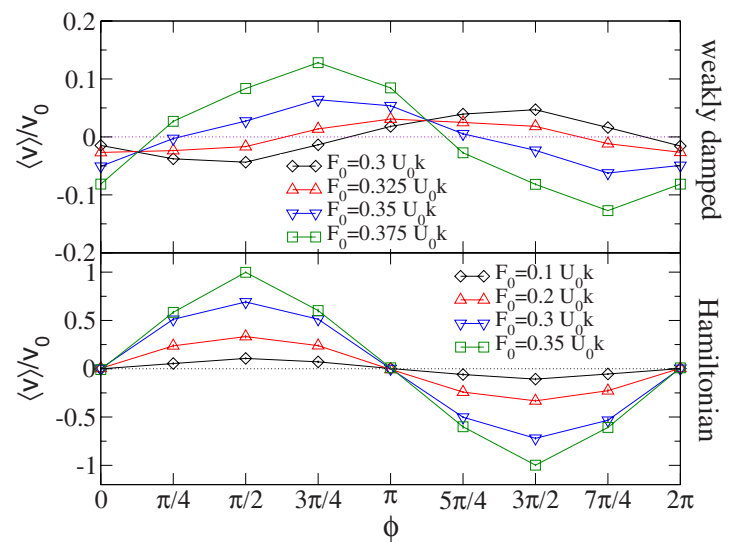

FIG. 1. (Color online) Average atomic velocity as a function of the relative phase $\phi$ between harmonics of the ac drive for several values of the driving amplitude $F_{0}$ and $\omega=\omega_{v}=k\left(2 U_{0} / m\right)^{1 / 2}$. Top panel: simulation data in the weakly damped regime. The friction and the noise strength values are fixed to $\alpha=0.15 \alpha_{0}$ and $D$ $=1.944 D_{0}$, respectively, where $\alpha_{0}=m k v_{0}, D_{0}=\alpha_{0}^{2} v_{0} / k$, and $v_{0}$ $=\left(U_{0} / m\right)^{1 / 2} / 10$. The values near $\phi=\pi / 2($ or $\phi=3 \pi / 2)$ show a current reversal as the driving amplitude is increased. Bottom panel: simulation data in the Hamiltonian regime $(\alpha=D=0)$. The initial conditions were chosen within the chaotic sea shown in Fig. 3. Lines are a guide to the eye.

is the overdamped regime. In this limit, the so-called "supersymmetry" [15] $(x, p, t) \rightarrow(x+\lambda / 2,-p,-t)$, with $\lambda$ as the spatial period of the potential, is satisfied for $\phi=\pi / 2+n \pi$, with $n$ as integer. For these values of the driving phase $\phi$ no directed transport can occur.

In order to establish a link between current reversals and symmetry breaking, we examine the dependence of the particles' current on two different quantities. First, we study the current as a function of the driving phase $\phi$, which controls the time symmetry of the Hamiltonian. This allows us to reveal the role of dissipation-induced symmetry breaking. Second, we consider the standard setup for the observation of current reversals: we fix the Hamiltonian by choosing a value of $\phi$ which corresponds to broken time-reversal symmetry $(\phi=\pi / 2$, say) and study the current as a function of the driving strength. This will allow us to detect current reversals and relate them to dissipation-induced symmetry breaking. In all the results presented in this work, the relative weight between the harmonics of the force is fixed $(A=B$ $=1$ in all numerical simulations) and we vary the overall amplitude $F_{0}$.

We examine first the weakly damped regime. The top panel of Fig. 1 shows the average particles' velocity as a function of the driving phase for different values of the amplitude of the drive. The displacement of the current curves' extrema clearly indicates that, for a given dissipation level (i.e., a given value of the friction coefficient $\alpha$ ), a variation of the drive amplitude leads to a variation in the dissipationinduced phase lag $\phi_{0}$. This is the central point of our analysis: because of dissipation, the curve of the average velocity vs the phase $\phi$ acquires a nonzero phase lag $\phi_{0}$, and the magnitude of the phase lag is a function of the strength of the driving. If we now examine the behavior of the current for a fixed phase $\phi$ (say, $\phi=\pi / 2)$ we see that the variation of $\phi_{0}$

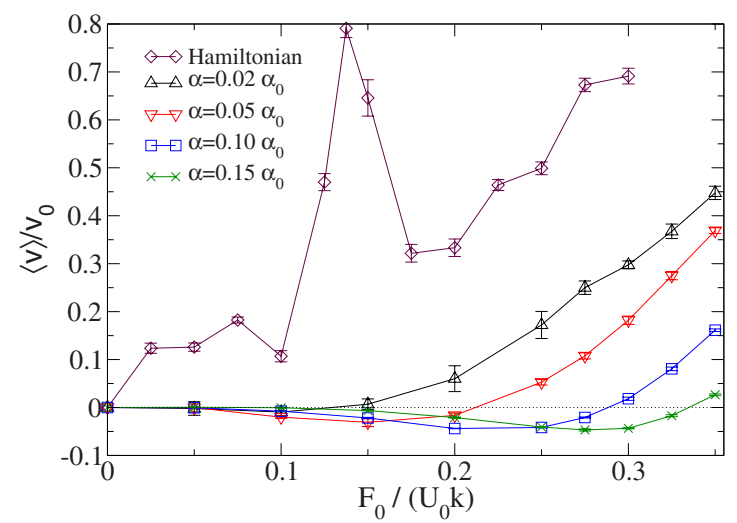

FIG. 2. (Color online) Simulation data in the weakly damped regime: current as a function of the driving amplitude $F_{0}$ for several values of the friction $\alpha$ and a fixed driving phase $\phi=\pi / 2$; rest of the parameters as in Fig. 1. As the dissipation is decreased, the current reversal position is shifted to lower values of the driving amplitude $F_{0}$. The diamonds show the results for the Hamiltonian system. Lines are a guide to the eye.

due to the change in driving strength leads to a current reversal. Thus, a link between current reversals and dissipation-induced symmetry breaking is established.

Our argument relies on the existence of a dissipationinduced symmetry-breaking phase lag $\phi_{0}$, whose value depends on the dissipation level and driving strength. The consistency of the argument can be verified by considering two extreme limits: the Hamiltonian case and the overdamped regime. In both cases, the phase lag $\phi_{0}$ is "locked" to a given value (zero for the Hamiltonian case and $\pi / 2$ in the overdamped regime) by the system symmetry and cannot be varied by modifying the driving strength. According to our argument, current reversals should disappear in both limits.

We consider the dissipationless limit. The bottom panel of Fig. 1 shows the average particles' velocity as a function of the phase $\phi$ for different values of the driving strength. As in the weakly damped case, the current shows a sinelike dependence on the phase $\phi$. However, unlike the weakly damped case, the zeros of the current curves are now fixed by the time-reversal symmetry, and a change in the driving strength does not induce any phase shift. If we now consider the current dependence on the driving strength $F_{0}$ for a given phase (say, $\phi=\pi / 2$ ), we observe that no current reversal occurs. These results are consistent with the link we established between current reversals and dissipation-induced symmetry breaking.

It is interesting to study the dynamics of disappearance of current reversals while approaching the Hamiltonian limit. This is done in Fig. 2, where for a fixed phase $\phi=\pi / 2$ the current is studied as a function of the driving strength, for different levels of dissipation. It appears that by decreasing dissipation, the position of the current reversal moves toward $F_{0}=0$, and in the Hamiltonian limit the current reversal disappears as its position coincides with $F_{0}=0$.

We notice that in the Hamiltonian limit the asymptotic particles' velocity depends on the initial preparation [22]. For the specific system of interest here, such a dependence is summarized in Fig. 3. There, we also evidence the chaotic 


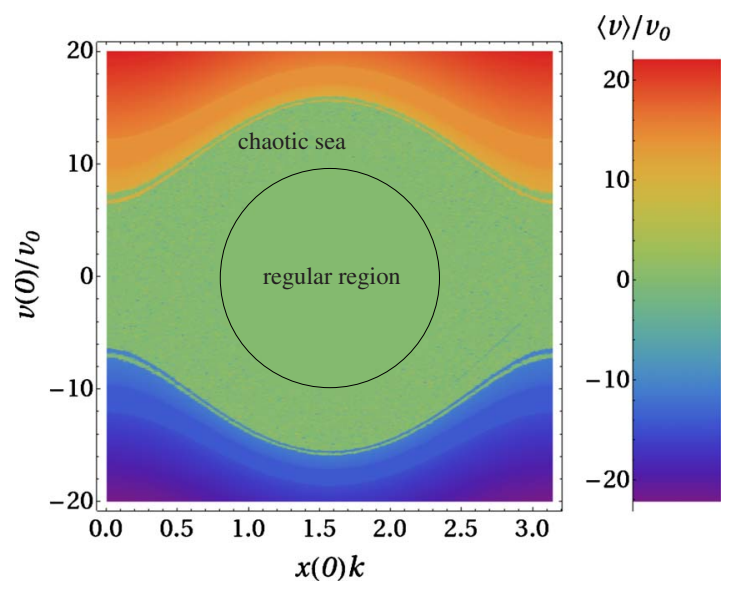

FIG. 3. (Color online) Simulation data in the Hamiltonian regime: the current as a function of the initial conditions $v(0)$ and $x(0)$ is represented using a color density plot. The driving parameters are $\omega=\omega_{v}, F_{0}=0.1 U_{0} k$, and $\phi=\pi / 2$. A black circumference indicates the boundary of a regular circular region in which the current is zero throughout.

sea. It is within that region where the initial conditions for the calculations in the Hamiltonian limit presented here (bottom panel of Fig. 1 and one set in Fig. 2) were chosen.

We now consider the overdamped case. Numerical simulations for this regime, in the presence of noise, are reported in the top panel of Fig. 4. The current is still described by a sinelike function $v \sim \sin \left(\phi-\phi_{0}\right)$, but the value of the phase lag is now locked by the symmetry to $\phi_{0}=\pi / 2$. A variation in the strength of the driving does not lead to a change in the phase lag. Studying the current as a function of the driving strength, for a fixed value $\phi=\pi / 2$ of the driving phase, reveals the absence of current reversals. This confirms our statement about a link between current reversals and the

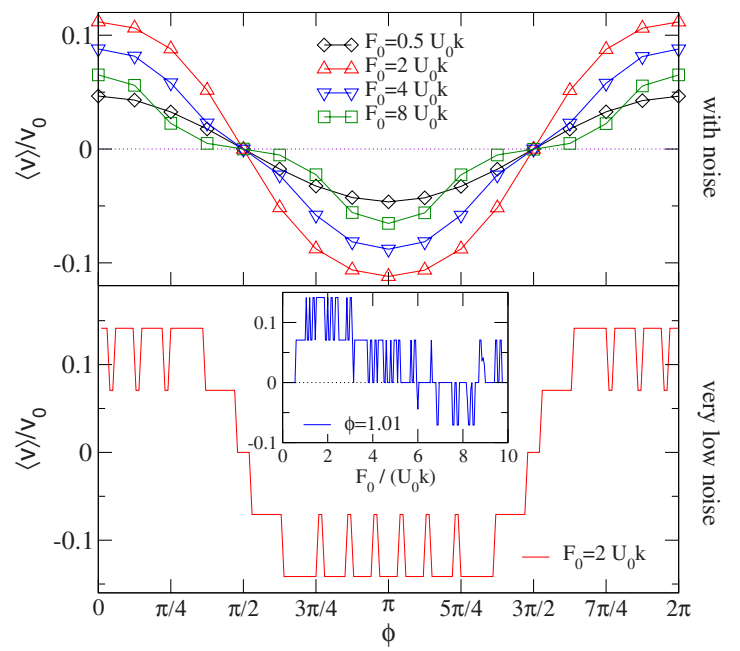

FIG. 4. (Color online) Simulation data in the overdamped regime: current as a function of the driving phase $\phi$ for several values of the driving amplitude $F_{0}$, with $\omega=0.01 \omega_{v}$ and a friction $\alpha$ $=100 \alpha_{0}$. Top panel: $D=1.944 \times 10^{3} D_{0}$, with lines being a guide to the eye. Bottom panel: $D=0.1944 \times 10^{-3} D_{0}$. The inset in the bottom panel shows the current as a function of $F_{0}$ for a fixed driving phase $\phi=1.01$, displaying multiple current reversals.
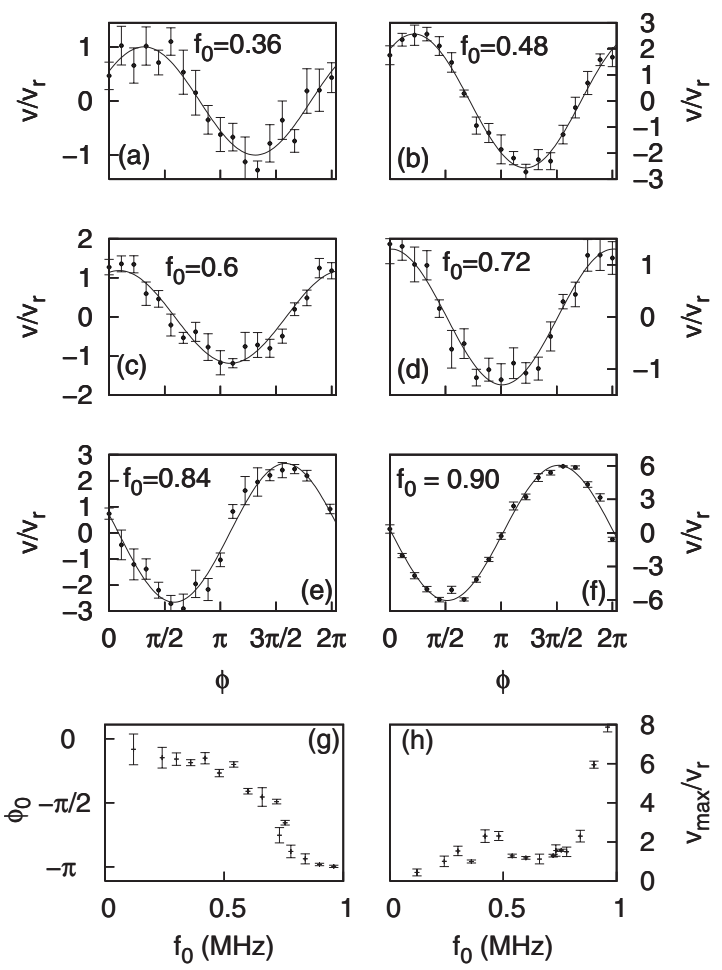

FIG. 5. Experimental results for 1D rocking ratchet for cold atoms. (a)-(f) Average atomic velocity, rescaled by the recoil velocity $v_{r}\left(v_{r}=5.88 \mathrm{~mm} / \mathrm{s}\right.$ for $\left.{ }^{87} \mathrm{Rb}\right)$, as a function of the relative phase $\phi$ between harmonics of the ac drive, for different values of the driving force amplitude. (g) Dissipation-induced phase lag $\phi_{0}$, as obtained by fitting data as those in (a)-(f) with the function $v / v_{r}$ $=A \sin \left(\phi-\phi_{0}\right)$, as a function of the driving amplitude. The amplitude $A$ is reported in (h). The parameters of the optical lattice are detuning from resonance $\Delta=-15 \Gamma$ and intensity per lattice beam $I_{L}=105 \mathrm{~mW} / \mathrm{cm}^{2}$. The rocking force is of the form of Eq. (2), with $\omega /(2 \pi)=100 \mathrm{kHz}, A=1, B=2$, and $F_{0}=-m f_{0} \omega / k$, where $m$ is the atomic mass, $k$ is the laser field wave vector, and the values of $f_{0}$ (in $\mathrm{MHz}$ ) for the different sets of data are reported in the figures.

symmetry breaking induced by the presence of a moderate amount of dissipation.

So far we have demonstrated that the dependence of the dissipation-induced phase lag $\phi_{0}$ on the driving strength $F_{0}$ results in current reversals, as observed by monitoring the dependence of the current at a fixed driving phase on the strength of the drive. For the same argument, this type of current reversals is absent in the Hamiltonian and overdamped regimes, where the phase lag $\phi_{0}$ does not vary with the amplitude of the drive. It is important to specify the regime of applicability of such reasoning, and underline that not all current reversals may be necessarily traced back to dissipation-induced symmetry breaking. In fact our argument relies on the assumption that the current can be well described by a smooth sinelike function $v \sim \sin \left(\phi-\phi_{0}\right)$. This holds provided that the noise level is sufficiently large to smooth the curve. Thus, at low noise levels additional current reversals may appear, not related to dissipation-induced symmetry breaking. An example of this is provided in the bottom panel of Fig. 4, where current reversals are observed in the overdamped regime at a very low level of noise. 
The weakly damped regime, of central interest here, can be explored using dissipative cold atoms ratchets [23]. We used a one-dimensional (1D) rocking ratchet setup for ${ }^{87} \mathrm{Rb}$ atoms [24], which corresponds to a spatially symmetric potential and a biharmonic rocking force of the form of Eq. (2). Proceeding along the lines of the theoretical analysis, we measured the atomic average velocity as a function of the driving phase $\phi$, for a given dissipation level and for different strengths of the driving. Our results, shown in Fig. 5, confirm the prediction of the general theory we presented. An increase in the applied driving force amplitude $F_{0}$ leads to a large variation in the phase lag $\phi_{0}$, as also summarized in Fig. 5(g). Also the amplitude of the curve varies with the driving amplitude $F_{0}$. However, it never becomes zero [see Fig. 5(h)]. It is thus the variation in the phase lag $\phi_{0}$ which produces a change in sign of the current observed for $\phi$ $=\pi / 2$, i.e., a current reversal.

In conclusion, in this work we studied, both theoretically and experimentally, the relationship between current reversals and symmetry breaking. For the specific system with a spatially symmetric potential and a time-asymmetric drive, we showed that a class of current reversals is actually determined by dissipation-induced symmetry breaking. As a consequence, these reversals are not present in the Hamiltonian limit or in the overdamped limit.

This research was supported by the Leverhulme Trust. One of us (D.C.) also thanks the Ministerio de Ciencia e Innovación of Spain for financial support (Grant No. FIS2008-02873).
[1] A. Ajdari and J. Prost, C. R. Acad. Sci., Ser. II: Mec., Phys., Chim., Sci. Terre Univers 315, 1635 (1992).

[2] M. O. Magnasco, Phys. Rev. Lett. 71, 1477 (1993).

[3] A. Ajdari, D. Mukamel, L. Peliti, and J. Prost, J. Phys. I 4, 1551 (1994).

[4] R. Bartussek, P. Hänggi, and J. G. Kissner, EPL 28, 459 (1994).

[5] C. R. Doering, W. Horsthemke, and J. Riordan, Phys. Rev. Lett. 72, 2984 (1994).

[6] P. Reimann, Phys. Rep. 361, 57 (2002).

[7] P. Hänggi and F. Marchesoni, Rev. Mod. Phys. 81, 387 (2009).

[8] F. Marchesoni, Phys. Lett. A 119, 221 (1986).

[9] M. C. Mahato and A. M. Jayannavar, Phys. Lett. A 209, 21 (1995).

[10] D. R. Chialvo and M. M. Millonas, Phys. Lett. A 209, 26 (1995).

[11] M. I. Dykman, H. Rabitz, V. N. Smelyanskiy, and B. E. Vugmeister, Phys. Rev. Lett. 79, 1178 (1997).

[12] I. Goychuk and P. Hänggi, EPL 43, 503 (1998).

[13] S. Flach, O. Yevtushenko, and Y. Zolotaryuk, Phys. Rev. Lett.
84, 2358 (2000).

[14] O. Yevtushenko, S. Flach, Y. Zolotaryuk, and A. A. Ovchinnikov, EPL 54, 141 (2001).

[15] P. Reimann, Phys. Rev. Lett. 86, 4992 (2001).

[16] L. Machura, M. Kostur, and J. Luczka, Chem. Phys. 375, 445 (2010).

[17] P. Jung, J. G. Kissner, and P. Hänggi, Phys. Rev. Lett. 76, 3436 (1996).

[18] J. L. Mateos, Phys. Rev. Lett. 84, 258 (2000).

[19] P. H. Jones, M. Goonasekera, and F. Renzoni, Phys. Rev. Lett. 93, 073904 (2004).

[20] To avoid a dependence of the current with the initial conditions, the biharmonic driving is switched on adiabatically both in the simulations and the experiments.

[21] N. R. Quintero, J. A. Cuesta, and R. Alvarez-Nodarse, Phys. Rev. E 81, 030102(R) (2010).

[22] H. Schanz, M.-F. Otto, R. Ketzmerick, and T. Dittrich, Phys. Rev. Lett. 87, 070601 (2001).

[23] M. Brown and F. Renzoni, Phys. Rev. A 77, 033405 (2008).

[24] F. Renzoni, Adv. At., Mol., Opt. Phys. 57, 1 (2009). 